\title{
The universal wave function interpretation of string theory
}

\author{
Dr. Zhi Gang Sha ${ }^{1}$, and Rulin $\mathrm{Xiu}^{2 \mathrm{a}}$ \\ ${ }^{1}$ Institute of Soul Healing and Enlightenment, 30 Wertheim Court, Unit 27D, Richmond Hill, Ontario L4B 1B9, \\ Canada \\ ${ }^{2}$ Hawaii Theoretical Physics Research Center, 16-266 E. Kipimana St, Keaau, HI 96749, USA
}

\begin{abstract}
In this work, we will show that a deeper understanding of space-time provided by both quantum physics and general relativity can lead to a new way to understand string theory. This new way of understanding and applying string theory, the universal wave function interpretation of string theory (UWFIST), may yield to a more powerful string theory and testable prediction. We will show how to derive UWFIST and what new result we can obtain from UWFIST. We will demonstrate that UWFIST indicates that the observed space-time and all phenomena are the projections from the world-sheet hologram. UWFIST provides the possible source for dark energy and dark matter and the explanation about why the dark energy and dark matter is beyond the detection of our current detector. We will show that UWFIST may also yield correct prediction of the cosmological constant to be of the order 10-121 in the unit of Planck scale. It may also help us understand and derive the energy source for inflation and the flatness of our observed 4-dimensional universe. UWFIST may also make other testable predictions that may be detected by interferometers.

We conclude that UWFIST has the potential to make string theory a more powerful physics theory that can yield testable predictions. It is worth further investigation by more physicists.
\end{abstract}

Quantum physics has been proven to be the fundamental physics for all the elementary particles and three fundamental forces, electromagnetic, weak and strong forces. General Relativity is the proven theory for gravity. Many ways are proposed to incorporate gravity and general relativity into quantum physics. String theory is so far the only mathematically consistent theory for quantum gravity (Ref $1,2)$.

How to understand and apply String Theory has been a long debated subject. The currently accepted interpretation is that string theory studies the quantum dynamics of a string. The vibration of string creates particles and interactions. The scale of the string is set to be Planck Scale. The finite size of the string length helps result in a consistent quantum gravity and gauge theory. The achievement of string theory cannot be over estimated. This theory has the potential to predict the dimension of space-time, the particle and interaction spectrum, to unify all the forces and fundamental particles, and more. However, with all these great potentials and promises, string theory has not yet made a single testable prediction. Something is still missing in our understanding of string theory.

In this work, we will show a different way to derive string theory. We will show that the deeper understanding of space-time can lead to a new way to understand string theory. This new way of

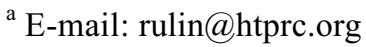


understanding and applying string theory may yield to a more powerful string theory and testable prediction.

In this paper, we will first derive the uncertainty relation between space-time. Then we use spacetime uncertainty relation to derive a string action. We will show that this provides a new way to understand string theory. Here we call it the universal wave function interpretation of string theory (UWFIST). We will discuss the possible testable predictions we can obtain from UWFIST. We will demonstrate that UWFIST indicates the possible source of dark energy and dark matter and provides explanation about why the dark energy and dark matter is beyond the detection of our current detectors. It also has the potential to give us the right estimate about cosmological constant as well as explain the energy source for the inflation. It may also unriddle why our observed 4-dimensional space-time is flat in the most part.

\section{Derivation of Uncertainty Relation Between Space and Time}

Quantum physics has completely changed our views about space-time in a way even more dramatic than the way Einstein has changed our view about space-time. According to quantum physics, how accurate we can measure time and space depends on the amount of energy and momentum we use. It takes energy and momentum to measure time and space. More specifically, according to quantum physics, to measure the time duration $\Delta \tau$, it takes the energy $\Delta \mathrm{E} \sim \mathrm{h} / \Delta \tau$. Similarly, to measure space size $\Delta \sigma$, it takes the momentum $\Delta \mathrm{P} \sim \hbar / \Delta \sigma$. Here $\hbar$ is the reduced Planck Constant, $\hbar=h / 2 \pi$. And $\mathrm{h}$ is the Planck Constant. It is equal to $6.626 \times 10^{-34}$ joul $\bullet$ second.

If one takes gravity into consideration, when one measures time interval $\Delta \tau$, the energy $\Delta \mathrm{E}$ used for time measurement will curve the space-time according to general relativity. It will create a black hole with the horizon on the order of $\mathrm{G} \Delta \mathrm{E} / \mathrm{c}^{4}$. Here $\mathrm{G}$ is the gravitational constant with the value approximately $6.674 \times 10^{-} 11 \mathrm{~N} \cdot \mathrm{m}^{2} / \mathrm{kg}^{2}$ and $\mathrm{c}$ is the speed of light with the value approximately $3 \times 10^{8}$ meter/second. When $\Delta \sigma$ is smaller than $\mathrm{G} \Delta \mathrm{E} / \mathrm{c}^{4}$, no information can come out. Therefore, the measurable causal region is:

$$
\Delta \sigma \geq G \Delta E / c^{4}
$$

This indicates an uncertainty relation between space $\Delta \sigma$ and time $\Delta \tau$ :

$$
\Delta \sigma \Delta \tau \geq l_{p} t_{p}
$$

Here $1_{p}$ is the Planck length, $1_{p}=\left(\hbar G / c^{3}\right)^{1 / 2}=1.616 \times 10^{-35}$ meter. And $t_{p}$ is the Planck time, $t_{p}=$ $\left(\hbar \mathrm{G} / \mathrm{c}^{5}\right)^{1 / 2}=5.39 \times 10^{-44}$ second.

The requirement (1) is the condition for the causal region. We call the relation (1) an innate uncertainty relation between space and time. It comes about when we take both quantum effect and gravity into account.

\section{A new way to derive and understand string theory}

The space-time uncertainty relation (1) indicates that space and time are quantized. With the spacetime uncertainty relation (1), there are two ways to quantize space and time in quantum mechanics.

In Quantum Physics, the uncertainty relations between time $\tau$ and energy $\mathrm{H}$ 


$$
\Delta H \Delta \tau \geq \hbar
$$

indicates the non-commutation relationship between time and $\mathrm{H}$

$$
[H, \tau]=i \hbar
$$

The uncertainty relationship between space $\sigma$ and momentum $P$

$$
\Delta P \Delta \sigma \geq \hbar
$$

indicates the non-commutation relationship between $\sigma$ and $\mathrm{P}$

$$
[P, \sigma]=i \hbar
$$

Using the same argument, we can see that the uncertainty relation (1) indicates the non-commutation relation between space and time:

$$
[\sigma, \tau]=i l_{p} t_{p}
$$

The first way to quantize space and time is to consider space and time as operators and they do not commute with each other $[\sigma, \tau]=\mathrm{i} 1_{\mathrm{p}} \mathrm{t}_{\mathrm{p}}$. This will lead to non-commutative space-time geometry. Many people have been exploring and studying physics with non-commutative space time geometry hoping to find a consistent theory for quantum gravity.

Here we are going to explore the second way to quantize space and time. In quantum physics, the uncertainty relation $\Delta \mathrm{H} \Delta \tau \geq \hbar$ and the non-commutation relationship between time and $\mathrm{H},[\mathrm{H}, \tau]=\mathrm{i}$ indicates an action $A_{t}$ in the form

$$
A_{t}=i \int_{0}^{t} H d \tau / \hbar
$$

This action $\mathrm{A}_{\mathrm{t}}$ will evolve wave function $\Psi$ from $\Psi(0)$ at the time 0 to $\Psi(\mathrm{t})$ at the time $\mathrm{t}$ in the form:

$$
\Psi(t)=\exp \left(i \int_{0}^{t} H d \tau / \hbar\right) \Psi(0) .
$$

In the same way, the uncertainty relation $\Delta \mathrm{P} \Delta \sigma \geq \hbar$ and the non-commutation relation between space coordinate $\sigma$ and momentum $P,[P, \sigma]=i \hbar$, indicates an action $A_{x}$ in the form

$$
A_{x}=i \int_{0}^{x} P d \sigma / \hbar
$$

This action $\mathrm{A}_{\mathrm{x}}$ will evolve wave function $\Psi$ from $\Psi(0)$ at the space 0 to $\Psi(\mathrm{x})$ at the space $\mathrm{x}$ in the form:

$$
\Psi(x)=\exp \left(i \int_{0}^{x} P d \sigma / \hbar\right) \Psi(0)
$$

Using the same quantization procedure, we propose that the space time uncertainty relation (1) and non-commutation relation (2) indicates an action $\mathrm{A}_{\mathrm{s}}$ in the form:

$$
A_{s}=\int d \tau d \sigma / l_{p} t_{p}
$$


This action $\mathrm{A}_{\mathrm{s}}$ evolves the wave function from $\psi(0,0)$ at the space 0 and time 0 to $\psi(\mathrm{L}, \mathrm{T})$ at the space $\mathrm{L}$ and time $\mathrm{T}$ in the following way:

$$
\psi(L, T)=\exp \left(i \int_{0}^{T} d \tau \int_{0}^{L} d \sigma / l_{p} t_{p}\right) \psi(0,0)
$$

It is easy to notice that action (3) is a string action (Ref 1,2). This demonstrates from another angle that when we take gravity into consideration, it is necessary to use a "string" action (3) instead of "particle" action $\mathrm{A}=\int \mathrm{d} \tau$ to obtain a consistent theory.

We will call the string theory expressed in the formula (4) the universal wave function interpretation of string theory. This universal wave function interpretation of string theory deviates from the usual interpretation of string theory in two ways. Firstly, the world-sheet space and time integration of the action (3) is over the causal region. i.e.

$$
A_{s}=\int_{0}^{T} d \tau \int_{0}^{L} d \sigma / l_{p}^{2}
$$

Here $\mathrm{T}$ and $\mathrm{L}$ are the age and length of the causal horizon. In the usual interpretation of string theory, $\sigma$ is the string coordinate. $\mathrm{L}$ is taken to be the length of the string. It is set to be Planck scale. In the formula (4), $\sigma$ and $\tau$ represent proper space and time. $T$ and $L$ are now variables. They change as universe evolves or as space and time pass by.

The second difference between the usual string theory and the string theory expressed in the equation (4) is that the universal wave function $\Psi(\mathrm{L}, \mathrm{T})$ are introduced. Here $\Psi(0,0)$ and $\Psi(\mathrm{L}, \mathrm{T})$ represents the universal wave function at the initial proper space and time and at the proper space $\mathrm{L}$ and time $\mathrm{T}$.

We call $\Psi(\mathrm{L}, \mathrm{T})$ the universal wave function for a good reason. For example, if we extend the string action to the cases of superstring and heterotic string with background fields created in the proper space-time, the universal wave function $\Psi$ can include all fundamental particles, all gauge interactions, gravity, and more. It also includes the large structures of our universe up to the scale of horizon. In other words, the universal wave function could be the wave function of our universe.

Except for these two major conceptual differences, the usual string theory calculation can still be applied here. We will call this new way to understand and apply string theory as Universal Wave Function Interpretation of String Theory (UWFIST). In the following, we will discuss what UWFIST can tell us about our universe.

\section{Our observed space-time and target space-time}

In UWFIST, the action (3) is in the proper space-time. The proper space and time $(\sigma, \tau)$ form the world sheet. The normal observed space-time is expressed by the normal space-time coordinate $X^{\mu}(\sigma, \tau)$, which is a projection from the proper space-time to the normal space-time. Because $X^{\mu}(\sigma, \tau)$ defines an embedding:

$$
\text { World-sheet -> Target Space, }
$$

the observed space-time is the target space. 
With the introduction of the target space-time coordinate $X^{\mu}(\sigma, \tau)$, the equation (4) becomes:

$$
A_{s}=\left(1 / l_{p} t_{p}\right) \int d \tau d \sigma g^{1 / 2} g^{a b} \boldsymbol{\partial} \boldsymbol{a} \boldsymbol{\mu} \boldsymbol{\partial} \boldsymbol{b} \boldsymbol{X}^{\boldsymbol{\mu}}
$$

Here $g^{\alpha \beta}$ is the metric tensor on the world-sheet formed by proper space and time $(\tau, \sigma)$ and $g=-$ det $\mathrm{g}^{\alpha \beta}$.

As shown in string theory (Ref 1,2), the general form of action (5) in the presence of massless background field is in the form:

$$
A_{s}=\left[i \alpha \int_{0}^{T} d \tau \int_{0}^{L} d \sigma g^{1 / 2}\left(g^{\alpha \beta} G^{\mu v} \partial_{\alpha} X_{\mu} \partial_{\beta} X_{v}+\varepsilon^{\alpha \beta} B^{\mu v} \partial_{\alpha} X_{\mu} \partial_{\beta} X_{v}+1 / 4 \alpha \Phi R\right)\right]
$$

Here $G^{\mu v}(\tau, \sigma), B^{\mu v}(\tau, \sigma), \Phi(\tau, \sigma)$ are metric tensor, anti-symmetric tensor, and scalar background fields of the target space. The target space-time coordinate $X^{\mu}(\tau, \sigma)$ is a vector projection from the proper space-time to the target space. The target space background fields $G^{\mu v}(\tau, \sigma), B^{\mu v}(\tau, \sigma)$, and $\Phi(\tau$, $\sigma)$ are various tensor projections from proper space and time.

Utilizing the Feynman Path Integral definition for the evolution of wave function as explained in Richard Feynman's work (Ref 3), the universal wave function in the presence of background fields now becomes:

$$
\begin{gathered}
\Psi\left(X^{\mu}(L, T), G^{\mu v}(L, T), B^{\mu v}(L, T), \Phi(L, T)\right)= \\
\int D X^{\mu} D g^{\mu v} D B^{\mu v} D \Phi \exp \left[i \alpha \int_{0}^{T} d \tau \int_{0}^{L} d \sigma g^{l / 2}\left(g^{\alpha \beta} G^{\mu v} \partial_{\alpha} X_{\mu} \partial_{\beta} X_{v}+\varepsilon^{\alpha \beta} B^{\mu v} \partial_{\alpha} X_{\mu} \partial_{\beta} X_{v}+1 / 4 \alpha \Phi R\right)\right]
\end{gathered}
$$

We can extend $\Psi\left(\mathrm{X}^{\mu}(\mathrm{L}, \mathrm{T}), \mathrm{G}^{\mu \nu}(\mathrm{L}, \mathrm{T}), \mathrm{B}^{\mu v}(\mathrm{~L}, \mathrm{~T}), \Phi(\mathrm{L}, \mathrm{T})\right)$ to include other massive vibrations. We can also extend the above string action to the cases of superstring and heterotic string to include all fundamental particles, all gauge interactions, gravity, and more. This universal wave function tells us the probability for our universe to be at space-time coordinate $\mathrm{X}^{\mu}(\mathrm{L}, \mathrm{T})$ and background fields $\mathrm{G}^{\mu v}(\mathrm{~L}, \mathrm{~T}), \mathrm{B}^{\mu v}(\mathrm{~L}, \mathrm{~T}), \Phi(\mathrm{L}, \mathrm{T})$. We can do a Fourier transformation to transform the wave function $\Psi\left(\mathrm{X}^{\mu}(\mathrm{L}, \mathrm{T}), \mathrm{G}^{\mu v}(\mathrm{~L}, \mathrm{~T}), \mathrm{B}^{\mu v}(\mathrm{~L}, \mathrm{~T}), \Phi(\mathrm{L}, \mathrm{T})\right)$ which are expressed in the target space represented by $\left.\mathrm{X}^{\mu}(\mathrm{L}, \mathrm{T}), \mathrm{G}^{\mu \nu}(\mathrm{L}, \mathrm{T}), \mathrm{B}^{\mu \nu}(\mathrm{L}, \mathrm{T}), \Phi(\mathrm{L}, \mathrm{T})\right)$ to the wave function $\Psi\left(\omega^{\mu}, \kappa^{\mu \nu}, \ldots\right)$ expressed in the vibrational space expressed by the various vibrations $\left(\omega^{\mu}, \kappa^{\mu v}, \ldots\right)$. The wave function $\Psi\left(\omega^{\mu}(\mathrm{L}, \mathrm{T}), \kappa^{\mu v}(\mathrm{~L}, \mathrm{~T}), \ldots\right)$ expresses what kind of vibrations in our universe and how much they exist at proper time $\mathrm{T}$ and horizon length $\mathrm{L}$ in our universe.

\section{The Existence of Dark Energy and Dark Matter}

A natural indication and consequence of UWFIST is the existence of dark energy and dark matter.

The space-time coordinate $X^{\mu}(\tau, \sigma)$ is composed of vibrations in the form $\exp [i \pi n(\sigma+c \tau) / L]$ and $\exp [i \pi n(\sigma-c \tau) / L]$. Here $n$ is an integer. Unlike the normal string theory, in which $\mathrm{L}$ is the Planck length, now $\mathrm{L}$ is causal horizon distance. The largest wavelength is on the order of the length of the causal horizon. These vibrations are very fine. They are very dark in the sense that it is very difficult to detect them. In fact, to detect the vibration with the wavelength $\mathrm{L}$, it takes the time $\mathrm{L} / \mathrm{c}$. If $\mathrm{L}$ is the horizon distance, this means that it takes the time of the age of the visible universe or a detector as large as the whole visible universe to detect these vibrations. The existence of these vibrations may explain the existence of dark energy. 
There also exist vibrations with small masses, which can be as small as $\mathrm{h} /\left(\mathrm{Tc}^{2}\right)$. These are extremely small masses. Our current detectors cannot detect these small massive particles. The existence of these vibrations with extremely small masses may explain the existence of dark matter.

We conclude that UWFIST indicates the existence of dark energy and dark matter. It also explains why the dark energy and dark matter is beyond the detection of our current detector.

\section{Hologram projection and equation of motion}

The proper space $\sigma$ and time $\tau$ form the world-sheet. The world-sheet has the Weyl symmetry, the local rescaling invariance of the world-sheet metric. The action (6) is invariant under the local rescaling of the world-sheet metric:

$$
\begin{gathered}
g^{\alpha \alpha \beta}(\tau, \sigma)=\exp (2 \omega(\tau, \sigma)) g^{\alpha \beta}(\tau, \sigma), \\
X^{\prime} \mu(\tau, \sigma)=X \mu(\tau, \sigma), \\
G^{\prime \mu v}(\tau, \sigma)=G^{\mu v}(\tau, \sigma), \\
B^{\prime \mu v}(\tau, \sigma)=B^{\mu v}(\tau, \sigma), \\
\Phi^{\prime}(\tau, \sigma)=\Phi(\tau, \sigma),
\end{gathered}
$$

for arbitrary $\omega(\tau, \sigma)$.

Weyl invariance of world-sheet indicates that if we expand or contract the world-sheet, the worldsheet will look the same. In other words, the world-sheet is a hologram. Our observed space-time $\mathrm{X}^{\mu}(\tau, \sigma)$, particles, gauge and gravity interactions are the projection from this hologram. In other words, the observed universe is a projection from the world-sheet hologram.

The fact that our observed space-time is a projection from a hologram has great consequences. We believe many result can be obtained from this fact. Here we will just point out a few of such results.

One of the results we can derive from the fact that our observed universe is a projection from the world-sheet hologram is the classical equation of motion regarding the background field $\mathrm{G}^{\mu \nu}, \mathrm{B}^{\mu \nu}, \Phi$, and more expanded as the functions of $X^{\mu}(\operatorname{Ref} 1,2)$. The detailed derivation is presented in the reference $(1,2)$. In the nutshell, it is obtained by calculating the higher order corrections to the action (6). The Weyl invariance is automatically preserved at the first order. However, the higher order corrections threaten to violate it. Requiring the preservation of Weyl Invariance at the higher orders leads to the classical equation of motion for the background fields $\mathrm{G}^{\mu \nu}, \mathrm{B}^{\mu \nu}$, and $\Phi$. These classical equations of motion include Einstein's general relativity. It is the natural extension of general relativity because they naturally include gauge interactions, different particles, and other background fields and matters.

It is interesting to see that our universal wave function interpretation of string theory includes the characteristics of both quantum physics and Einstein's general relativity. Both the wave function and equation of motion come out of it naturally. It unifies quantum physics and Einstein's general relativity in such a fascinating way.

\section{World-Sheet Contains All The Information of Our Universe}

In the universal wave function interpretation of string theory, as we have discussed above, the worldsheet is the hologram from which our observed universe is projected. Because our observed universe is the projection from the world-sheet, the world sheet contains all the information about our universe. In the following, we will demonstrate this explicitly. 
There are demonstrations in black hole physics and strong evidence that the area of any surface limits the information content of adjacent space-time regions at $1.4 \times 10^{69}$ bits per square meter. This is often called Holographic principle (Ref 4,5,6,7,8,9). If we consider $\mathrm{T}$ and $\mathrm{L}$ as the time length and distance of the horizon of our universe, from the Holographic principle, the maximum amount of information in our universe should be proportional to TL.

Now we will demonstrate that the observable information created on the world-sheet $(\mathrm{T}, \mathrm{L})$ is also proportional to TL. Firstly, we will show that the space-time uncertainty relation indicates that there exist only finite observable states on the world sheet.

The smallest energy and momentum it takes to measure time duration $\Delta \tau$ and space length $\Delta \sigma$ is on the order of: $\Delta \mathrm{E} \Delta \tau \sim \hbar, \Delta \mathrm{E} \sim \hbar / \Delta \tau$, and $\Delta \mathrm{p} \Delta \sigma \sim \hbar, \Delta \mathrm{p} \sim \hbar / \Delta \sigma$, and $\Delta \sigma \Delta \tau \geq 1_{\mathrm{p}} \mathrm{t}_{\mathrm{p}}$, thus

$$
\Delta E \Delta p \sim \hbar^{2} /(\Delta \sigma \Delta \tau) \leq c \hbar^{2} /\left(l_{p}^{2}\right) .
$$

Since $\Delta \mathrm{E}=\hbar \omega, \Delta \mathrm{p}=\hbar \mathrm{k}, \omega$ and $\mathrm{k}$ relate to the frequency and wavelength of the vibrations used in the measurement, we obtain:

$$
\omega k \leq c / l_{p}^{2}
$$

Since $\omega=\mathrm{n} / \mathrm{T}, \mathrm{n}=1,2, \ldots$, and $\mathrm{k}=\mathrm{m} / \mathrm{L}, \mathrm{m}=1,2, \ldots$, we have:

$$
\begin{gathered}
\omega k=n m /(T L) \leq c /\left(l_{p}^{2}\right), \\
n m \leq c T L /\left(l_{p}^{2}\right)
\end{gathered}
$$

Therefore there are maximum TLc/ $1_{p}^{2}$ number of energy states. Since each energy state has 2 vibrational states, corresponding to $\mathrm{e}^{\mathrm{iwt} \pm \mathrm{ikx}}$ states, the number of the total possible states is $2^{\wedge}(\mathrm{cTL} /$ $1_{\mathrm{p}}^{2}$ ). The amount of information is:

$$
\text { Information }=\log _{2}\left(2^{\wedge}\left(c T L / l_{p}^{2}\right)\right)=\left(c T L / l_{p}^{2}\right)
$$

We can see that the observable information on the world-sheet is also proportional to TL in Planck Units.

We conclude that world-sheet contains all the information of our universe.

\section{Calculation of Vacuum Energy and Its Implication For Inflation}

Now we are going to calculate the vacuum energy in our observed space-time. Here we define the vacuum energy to be the lowest energy state of the universal wave function. To calculate the vacuum energy, we need to calculate the wave function in formula (7). Here we will only make a simple estimate of the vacuum energy. We will discuss the detailed calculation in our future work.

Now let's assume that the energy is conserved in the process of the projection from world-sheet to our target space. Under this assumption, by calculating the vacuum energy of the world-sheet, we can obtain the amount of vacuum energy of our observed universe. In the following, we will calculate the vacuum energy of the world sheet. From that, we deduce the vacuum energy density of our observed universe and the cosmological constant. We will show that this calculation can lead to the prediction of cosmological constant consistent with the current experimental data. 
In the above, we have shown that there is finite number of possible energy states in the causal horizon. Here we assume supersymmetry is broken at the space and time scale $\mathrm{T}$ and $\mathrm{L}$. If the supersymmetry is preserved, the total vacuum energy remains zero because the contributions from the bosonic and fermionic part cancel out.

We further assume that on the world-sheet, the energy at the state $(n, m)$ with $\omega_{n}=n / T$ and $k_{m}=$ $\mathrm{m} / \mathrm{L}$ is in the form of a harmonic oscillator:

$$
E_{n, m}=(n+1 / 2) h / T \text {. }
$$

The lowest energy of vacuum fluctuation is $E_{0 n, m}=h / 2 T$.

The total lowest vacuum energy is:

$$
E_{v a c}=h / 2 T \times T L / l_{p}^{2}=h L /\left(2 l_{p}^{2}\right) .
$$

It is proportional to the length of the causal horizon.

If we assume the total vacuum energy of the world-sheet is the same as the total vacuum energy of target space, then we can use the total vacuum energy (8) to derive the vacuum energy density and cosmological constant of our universe. The lowest vacuum energy density in the four dimensional space-time target space is then:

$$
\rho_{v a c}=E_{v a c} /\left(4 \pi L^{3} / 3\right)=\rho_{p} l_{p}^{2} /\left(2 L^{2}\right) .
$$

Here $\rho_{\mathrm{p}}$ is the Planck energy density,

$$
\rho_{p}=E_{p} /\left(4 \pi l_{p}^{3} / 3\right)
$$

and $E_{p}$ is the Planck energy $E_{p}=\hbar / t_{p}, t_{p}$ and $l_{p}$ are the Planck time and Planck length $1_{p}=c t_{p}=$ $\left(\hbar G / c^{3}\right)^{1 / 2}$. The equation (9) tells us that the vacuum energy density observed in three space dimensions is inversely proportional to the square of the length of horizon $\mathrm{L}$.

From the result (8), we can predict that if the observed space is one dimension, the energy density is a constant $\mathrm{h} /\left(2 \mathrm{1}_{\mathrm{p}}{ }^{2}\right)$. In 2-dimensional observed space, the energy density is inversely proportional to the length of horizon L. In n-dimensional observed space, the energy density is proportional to $\mathrm{L}^{1-\mathrm{n}}$.

Our result (9) indicates the vacuum energy as the energy source for inflation as well as for the continuous expansion of our universe. It shows us that if our universe starts at the Planck scale $1_{p}$ at this initial state, the vacuum energy density is half of the Planck energy density $\rho_{\mathrm{vac}}=\rho_{\mathrm{p}} / 2$. This large vacuum energy density provides the energy source for inflation, the rapid initial expansion. As universe expands, the vacuum energy density decreases, but is non-zero. Therefore our universe will continue to expand but its expansion will slow down.

Now let us assume that $\mathrm{L}$ is the Hubble radius with $\mathrm{L}=\mathrm{c} / \mathrm{H}$. Here $\mathrm{c}$ is the speed of light and $\mathrm{H}$ is the Hubble constant. Hubble constant is the unit of measurement used to describe the expansion of the universe. It has also been used to estimate the age of our universe. With this assumption, we get:

$$
\rho_{\text {vac }}=E_{\text {vad }} /\left(4 \pi L^{3} / 3\right)=3 h /\left(8 \pi c L^{2} l_{p}^{2}\right)=3 c^{2} /\left(8 \pi G L^{2}\right)=3 H_{0}^{2} /(8 \pi G)=\rho_{c}
$$

Since the relation (10) is general for all $\mathrm{L}$ and $\mathrm{T}$, it shows that the lowest vacuum energy density is equal to the critical energy density for 4-dimensional space-time and remains to be so as universe expands. This may provide a possible way to explain why our universe's energy density is so close to 
critical energy density. It indicates our universe may start and remain to be naturally flat for the most part in the 4-dimensional observed space-time.

The result (10) can explain why our observed 4-dimensional space-time is at the critical density at the vacuum state. It also indicates that for the observed space-time other than 4 dimensions of spacetime, the vacuum energy density is not at the critical energy density. Could this be the reason why we can only observe 4-dimensional space-time?

The recent Wilkinson Microwave Anisotropy Probe (WMAP) measurements have led to the conclusion of a flat universe, i.e. our universe's energy density is of the value of critical energy density with only a $0.5 \%$ margin of error $[10,11,12,13,14,15]$.

Inflation is so far the mostly accepted way to explain the flatness of the universe and to solve the fine-tuning problems with the initial conditions of the universe. Inflation is the proposed rapid exponential expansion of the early universe by a factor of at least $10^{78}$ in volume, driven by a negative-pressure vacuum energy density [16]. Inflation hypothesis is proposed to account for why our universe appears flat, homogeneous, and isotropic and to explain the origin of the large-scale structure of the cosmos. The hypothetical particle or field thought to be responsible for inflation is called inflaton. However, the detailed particle physics mechanism responsible for inflation or inflaton remains unknown. Furthermore, the abundance of "bad inflation" in the quantum theory and the realization that inflation is eternal has been challenging the real predictive power of inflation theory $[17,18,19,20]$.

UWFIST provides a simple explanation and calculable prediction from the first principle about why our universe experiences fast inflation at the early stage, continue to expand, and at the same time remains flat as seen from the 4-dimensional space-time point of view. It further predicts that the observed space-time with dimensions other than four is not flat.

\section{Calculation of Cosmological Constant}

The non-zero cosmological constant was first indicated by the observations presented in 1998 of distance-red shift relation for Type Ia supernovae that the expansion of the universe is accelerating $[10,11]$. When combined with measurements of the cosmic microwave background radiation these data provided the first direct evidence that cosmological constant $\Lambda$ is non-zero, with $\Lambda \sim 1.7 \times 10^{-121}$ in Planck units. This result has been supported by more recent WMAP measurements independently. Although there are other possible causes of an accelerating universe, the cosmological constant appears to be the simplest solution.

There are many efforts to derive the value of the cosmological constant and to understand why the cosmological constant $\Lambda$ is of the magnitude of $10^{-121}$ in Planck units. One approach is to assume parallel universes. One uses anthropic selection and prior probability distribution for $\Lambda$ over the multiverse to find the most probable value that allows galaxies to form [21,22,23]. J.D. Barrow and D.J Shaw and other people make the cosmological constant into a field and try to determine the cosmological constant from the equation of motion [24].

Next we will estimate the value of the cosmological constant using the vacuum energy density we have calculated (9). We know that:

$$
l_{p}^{2} / 2 L^{2} \sim t_{p}^{2} / 2 t_{u}^{2} \sim 10^{-122},
$$

here $t_{u}$ is the age of the universe. Since the cosmological constant $\Lambda_{c}=8 \pi \rho_{v a c}$, we obtain: 


$$
\Lambda_{c}=4 \pi \rho_{p} t_{p}^{2} / t_{u}^{2} \sim 10^{-121} \rho_{p}
$$

It is quite amazing that in UWFIST, using the age of the universe and the fundamental constants $\hbar$ and $\mathrm{G}$ we are able to derive the value of cosmological constant to the same order of magnitude as the observed value.

\section{Testable String Theory}

It is also important to point out that UWFIST may be tested by experiment. From the string action in the presence of the background fields (5), we can see that space-time coordinates $X_{\mu}(\tau, \sigma)$ and gravity $\mathrm{G}^{\mu v}(\tau, \sigma)$ are quantum entangled. Further we can see that because the commutation relationship: $[\sigma, \tau]=\mathrm{i} 1_{\mathrm{p}} \mathrm{t}_{\mathrm{p}}$ and the observed target space coordinate $\mathrm{X}^{\mu}(\tau, \sigma)$ is the function of $\sigma$ and $\tau$, there exists a similar commutation relationship for $X^{\mu}(\tau, \sigma)$ at different directions.

Craig J. Hogan has pointed out that this Planckian scale commutation relationship may be detected by interferometers (Ref 25). This may provide a way to experimentally test UWFIST. We hope future research studies can further explore this.

\section{Multiverse}

It is interesting to notice that our wave function interpretation of String Theory supports the multiverse hypothesis (Ref 26, 27). The calculation of the universal wave function in the presence of background fields using equation (5) gives the expression of the possible universes.

Multiverse has been hypothesized in modern theoretical physics to solve many unsolved problems in cosmology. However, the mathematical expression of the multiverse is usually obtained in an arbitrary way. The structure of the multiverse, the nature of each universe within it and the relationships among the various constituent universes, depend on the specific multiverse hypothesis considered. In wave function interpretation of string theory, the possible multiverse can be calculated mathematically from string theory using the equation (5).

\section{Conclusion}

In this paper, we propose a new way, the universal wave function interpretation of string theory (UWFIST), to understand and apply string theory. From the derived space-time uncertainty relation, we show that string theory can be understood as calculating the evolution of universal wave function by the string action created by the proper space-time. Our observed universe is the projection from world-sheet hologram to target space. We demonstrate that this new understanding of string theory can lead to testable prediction from string theory. It can provide the calculation of dark energy and dark matter and understanding about why dark energy and dark matter are beyond our current detection. It may lead to the prediction of cosmological constant consistent with the experimental result. It may also show us the possible energy source for the inflation and expansion of our universe. It may also explain why our universe remains flat in 4-dimension space-time. UWFIST deserves further investigation by more astrophysicists and cosmologists.

UWFIST also makes string theory a more powerful physics theory. It may enable string theory to make testable predictions. It is worth further investigation by more string theorists. 


\section{Acknowledgements}

We want to thank professor Mary K Gaillard, Dr. Ervin Laszlo, and many others for their help, support and encouragement with this project. I want to thank Rasha Blais and Alan Chuck for editing this paper.

\section{References}

1. Michael Green, John H. Schwarz and Edward Witten Superstring theory. Cambridge University Press. Vol. 1: Introduction. ISBN 0-521-35752-7. Vol. 2: Loop amplitudes, anomalies and phenomenology. ISBN 0-521-35753-5 (1987).

2. Polchinski, Joseph String theory. Cambridge University Press. Vol. 1: An Introduction to the Bosonic String. ISBN 0-521-63303-6.Vol. 2: Superstring Theory and Beyond. ISBN 0-52163304-4 (1998).

3. Feynman, Richard P.; Hibbs, Albert. Quantum Mechanics and Path Integrals. McGraw Hill. ISBN 0-07-020650-3 (1965).

4. Bekenstein, Jacob D. "Universal upper bound on the entropy-to energy ratio for bounded systems". Physical Review D 23 (215): 287-298 (January 1981).

5. 't Hooft, Gerard Dimensional Reduction in Quantum Gravity. pp. 10026. arXiv:gr-qc/9310026. Bibcode 1993gr.qc....10026T (1993).

6. Don N. Page. Information in Black Hole Radiation, hep-th/9306083, Phys. 36 6377-6396 (1995)

7. Susskind, Leonard. "The World as a Hologram". Journal of Mathematical Physics 36 (11): $6377-$ 6396. arXiv:hep-th/9409089. Bibcode 1995JMP....36.6377S. doi:10.1063/1.531249 (1995)

8. 20. Majumdar, Parthasarathi. "Black Hole Entropy and Quantum Gravity". ArXiv: General Relativity and Quantum Cosmology 73: 147. arXiv:gr-qc/9807045. Bibcode 1999InJPB..73..147M (1998).

9. 21. Bousso, Raphael. "The holographic principle". Reviews of Modern Physics 74 (3): 825-874. arXiv:hep-th/0203101. Bibcode 2002RvMP...74..825B. doi:10.1103/RevModPhys.74.825 (2002).

10. Joanne C. Baker, Keith Grainge, M.P. Hobson, Michael E. Jones, R. Kneissl, A.N. Lasenby, C.M.M. O'Sullivan, Guy Pooley, G. Rocha, Richard Saunders, P.F. Scott, E.M. Waldram. "Observational Evidence from Supernovae for an Accelerating Universe and a Cosmological Constant". The Astronomical Journal 116 (3): 1009-1038. arXiv:astro-ph/9805201. Bibcode 1998AJ...116.1009R. doi:10.1086/300499 (September 1998).

11. S. Perlmutter, G. Aldering, G. Goldhaber, R.A. Knop, P. Nugent, P.G. Castro, S. Deustua, S. Fabbro, A. Goobar, D.E. Groom, I. M. Hook, A.G. Kim, M.Y. Kim, J.C. Lee, N.J. Nunes, R. Pain, C.R. Pennypacker, R. Quimby, C. Lidman, R.S. Ellis, M. Irwin, R.G. McMahon, P. RuizLapuente, N. Walton, B. Schaefer, B.J. Boyle, A.V. Filippenko, T. Matheson, A.S. Fruchter, N. Panagia, H.J.M. Newberg, W.J. Couch (The Supernova Cosmology Project). "Measurements of Omega and Lambda from 42 High-Redshift Supernovae". The Astrophysical Journal 517 (2): 565-586. arXiv:astro-ph/9812133. Bibcode 1999ApJ...517..565P. doi:10.1086/307221 (June 1999).

12. Bennett, C.; et al. "The Microwave Anisotropy Probe (MAP) Mission". Astrophysical Journal 583 (1): 1-23. arXiv:astro-ph/0301158. Bibcode 2003ApJ...583...1B. doi:10.1086/345346 (2003a). 
13. Bennett, C.; et al. "First-Year Wilkinson Microwave Anisotropy Probe (WMAP) Observations: Foreground Emission". Astrophysical Journal Supplement 148 (1): 97-117. arXiv:astroph/0302208. Bibcode 2003ApJS..148...97B. doi:10.1086/377252 (2003b).

14. Hinshaw, G.; et al. "Three-Year Wilkinson Microwave Anisotropy Probe (WMAP1) Observations: Temperature Analysis". Astrophysical Journal Supplement 170 (2): 288-334. arXiv:astro-ph/0603451. Bibcode 2007ApJS..170..288H. doi:10.1086/513698 (2007).

15. Hinshaw, G. et al. (WMAP Collaboration). "Five-Year Wilkinson Microwave Anisotropy Probe Observations: Data Processing, Sky Maps, and Basic Results". The Astrophysical Journal Supplement 180 (2): 225-245. arXiv:astro-ph/id=0803.0732. Bibcode 2009ApJS..180..225H. doi:10.1088/0067-0049/180/2/225. http://map.gsfc.nasa.gov/universe/uni_shape.html (Feb 2009).

16. Guth, Alan “The Inflationary Universe”. Basic Books (1998).

17. Steinhardt, Paul J. and Neil Turok "Endless Universe: Beyond the Big Bang." Doubleday (2007).

18. Physical Review Letters 59 (22): 2607-2610. Bibcode 1987PhRvL..59.2607W. doi:10.1103/PhysRevLett.59.2607. PMID 10035596.

19. Penrose, Roger. The Road to Reality: A Complete Guide to the Laws of the Universe. London: Vintage Books, p. 755 (2004).

20. Penrose, Roger "Difficulties with Inflationary Cosmology". Annals of the New York Academy of Sciences 271: 249-264. Bibcode:1989NYASA.571..249P. doi:10.1111/j.17496632.1989.tb50513 (1989).

21. Dicke, R. H. "Dirac's Cosmology and Mach's Principle". Nature 192 (4801): 440-441. Bibcode 1961Natur.192..440D. doi:10.1038/192440a0 (1961).

22. Weinberg, S. "Anthropic bound on the cosmological constant" (1987).

23. Steinhardt, Paul J. "The inflation debate: Is the theory at the heart of modern cosmology deeply flawed?" (Scientific American, April; pp. 18-25) (2011).

24. Barrow, John D. and Shaw, Douglas J. "The Value of the Cosmological Constant". General Relativity and Gravitation 43, 2555-2560 (2011). arXiv:1105.3105 [gr-qc] (May 2011).

25. Craig J. Hogan Interferometers as Probes of Planckian Quantum Geometry. arXiv:1002.4880 (2012)

26. Steinhardt, Paul J. "The inflation debate: Is the theory at the heart of modern cosmology deeply flawed?" (Scientific American, April; pp. 18-25) (2011).

27. Barrow, John D. and Shaw, Douglas J. "The Value of the Cosmological Constant". General Relativity and Gravitation 43, 2555-2560. arXiv:1105.3105 [gr-qc] (2011). 